\title{
Morphological features of the heart of six mosquito species as revealed by scanning electron microscopy
}

\author{
Gustavo Ferreira Martins ${ }^{1 *}$, José Marcelo Ramalho-Ortigão ${ }^{2}$ \\ and Paulo Filemon Paolucci Pimenta ${ }^{3}$ \\ ${ }^{1}$ Departamento de Biologia Geral, Universidade Federal de Viçosa, \\ DBG-UFV, CEP 36570-000 Viçosa, MG, Brazil, ²Department of \\ Entomology, Kansas State University (KSU), Manhattan, KS, USA and \\ ${ }^{3}$ Laboratório de Entomologia Médica, Instituto René Rachou - IRR, \\ Fundação Oswaldo Cruz (FIOCRUZ), Av. Augusto Lima, 1715, \\ CEP 30190-002 Belo Horizonte, MG, Brazil
}

(Accepted 18 May 2011)

\begin{abstract}
The circulatory system in insects is formed by a dorsal vessel (heart) that is a tubular structure that pumps haemolymph in the insect body cavity. Up until now, much is known about the structural organization of the heart in certain Brachycera (Diptera) (e.g. Drosophila). In contrast, little information is available regarding members of the Nematocera suborder (e.g. mosquitoes), in spite of their relevance to human health. Considering their importance, details of the heart morphology of six mosquito species (Aedes albopictus, Ae. aegypti, Ae. fluviatilis, Culex quinquefasciatus, Anopheles darlingi and An. aquasalis) were investigated by scanning electron microscopy (SEM). The heart of the adult mosquitoes, both males and females, consists of a dorsal tubular structure in association with pericardial cells located alongside the heart and alary muscles displaying ramifications that extend from the body wall to the heart surface. The SEM analysis also revealed in detail the organization of the heart, including paired valves on the heart wall (ostia). This study provides a detailed analysis of the mosquito heart microanatomy and, in our view, represents a significant contribution towards the understanding of structural-functional relationships associated with the heart of mosquito.
\end{abstract}

Key words: heart, pericardial cells, alary muscles, Aedes albopictus, Aedes aegypti, Aedes fluviatilis, Culex quinquefasciatus, Anopheles darlingi, Anopheles aquasalis

\section{Introduction}

Mosquitoes (Diptera: Culicidae) are the most important vectors of human diseases, being responsible for scourges such as malaria, dengue, filariasis and encephalitis. In spite of their impact on human health, much remains to be known in terms of the physiology and structural-functional relationships regarding mosquito tissues. Such knowledge gaps may, to some extent, be due to

*E-mail: gmartins@ufv.br the large number of species encompassing the various mosquito vectors (reviewed by Foster and Walker, 2009). An example of the current lack of information on mosquitoes regards the structural organization of the mosquito heart. The most recent exception is Anopheles gambiae Giles (Andereck et al., 2010; Glenn et al., 2010). Nevertheless, by and large, nothing is known about the heart organization for other mosquito species.

Insects display an open circulatory system consisting of a dorsal vessel that is a tubularshaped organ, located medially and dorsally on the 
body plan. The dorsal vessel is a muscular pulsate organ that extends from the head to the abdominal end and constitutes the main system through which haemolymph is pumped. The dorsal vessel is divided into two regions: a posterior abdominal heart with wall valves (ostia) and an anterior aorta that, in most studied insects, is a simple, imperforated tube (reviewed by Chapman, 1998).

The heart of the insect occurs in close association with alary muscles and pericardial cells. The alary muscle fibres are arranged in pairs and constitute the heart suspensory apparatus, while pericardial cells are found along the length of the dorsal vessel (Ejaz and Lange, 2008). The pericardial cells are believed to play a role in innate immune responses as phagocytic cells (Crossley, 1985; Miller, 1985; Curtis et al., 1999) and in homeostasis through the filtration of toxic substances from the haemolymph (Das et al., 2008; Weavers et al., 2009; Denholm and Skaer, 2009).

The dorsal vessels of the mosquitoes Aedes aegypti (L.) and Anopheles quadrimaculatus Say were described by Christophers (1960) and Jones (1954), respectively, and two recent studies have demonstrated various morphological and functional aspects of the An. gambiae heart (Andereck et al., 2010; Glenn et al., 2010). In this study, we applied the scanning electron microscopy (SEM) technique to investigate the structure of the heart of six adult mosquito species (Aedes albopictus [Skuse], Ae. aegypti [Linnaeus], Ae. fluviatilis [Lutz], Culex quinquefasciatus [Say], Anopheles darlingi [Root] and An. aquasalis [Curry]), including known major disease vectors. Our analyses revealed for the first time a general overview of the microanatomy of the heart of several mosquito species.

\section{Materials and methods}

In this study, 1-day-old male and female $A e$. albopictus, Ae. aegypti, Ae. fluviatilis, C. quinquefasciatus, An. darlingi and An. aquasalis were used. Except for An. darlingi, all other mosquitoes were obtained from colonies maintained at the Laboratory of Medical Entomology of the Instituto René Rachou, FIOCRUZ, Belo Horizonte, MG, Brazil. An. darlingi F1 were obtained from field-collected mosquitoes in and around the city of Manaus, AM, Brazil.

Mosquitoes were dissected under stereoscope in $1 \times$ phosphate-buffered saline (PBS), $\mathrm{pH}$ 7.2. The abdomen was separated from the thorax, cut laterally, and all the internal organs were removed. Approximately, $50 \mu \mathrm{l}$ of fixative solution containing $2.5 \%$ glutaraldehyde in $1 \times$ PBS supplemented with $7 \%$ sucrose was added directly to the abdominal dorsal part.

Fixed samples were post-fixed in 1\% osmium tetroxide in $0.8 \%$ potassium ferricyanide and
$0.1 \mathrm{M}$ cacodylate buffer, $\mathrm{pH}$ 7.2. Each sample was dehydrated in a crescent series of $70-100 \%$ acetone, dried in a critical point device using $\mathrm{CO}_{2}$ and goldcoated in a sputtering device for observation under the scanning electron microscope (JEOL JSM 5600).

\section{Results}

The results obtained revealed that, in general, the heart of the mosquito consists of a muscular tube located directly below the dorsal cuticle, running the length of the abdomen. The general organization of the heart of the mosquito species investigated in this study is provided in Fig. 1, depicting the heart of an An. darlingi female. Alary muscles occur in the seven anterior abdominal segments. These muscles attach individually (Fig. 1B) or in pairs (Fig. 2A) onto the two lateral sides of the heart and branch out in the form of ramifications or myofibrils throughout the length of the heart wall. Such attachment and branching out areas alternate with the pericardial cell being present at or near the points of attachment. Pericardial cells occur along the sides of the heart and are found within the ramifications of the alary muscles. Pericardial cells are also associated with the alary muscles in all abdominal segments. Interestingly, they are absent at the sites where the myofibrils branch out (Fig. 1).

Detailed views of mosquito heart ventral surfaces are shown in Figs $1 \mathrm{~B}$ and $\mathrm{C}$ (Ae. fluviatilis) and 2A (Ae. aegypti). Again, the results are representative of what was observed for all mosquito species investigated. Alary muscles are striated (Fig. 2B) and directly attached to the body wall, extending from the fat body to the heart surface (Fig. 1A and B). These muscles form a network, with branches resembling baskets that include pericardial cells. On both sides of the heart, these branches or ramifications anastomose above the heart surface (Figs 1C and 2A). Pericardial cells are mostly round cells located on the edges of the heart and organized in single lines on each side of the organ (Fig. 1A). In addition, portions of these cells extrude from the ramifications of alary fibre limits (Fig. 2A and C), and display surface deformations and invaginations (Fig. 2C). Segmental openings or ostia (Fig. 2A and inset) corresponding to heart valves were also detected. On preparations where the heart wall is eventually broken, muscle fibres can be seen circularly arranged, providing a view of the striated aspect of the interior of the heart wall (Fig. 2A).

\section{Discussion}

In our view, this study represents a comprehensive expansion of the data available to date on structural 
features of the mosquito heart. In spite of the importance of mosquitoes as vectors of human and animal pathogens, and the significant amount of information on various details of the physiology and tissue/organ organization in mosquitoes, the same cannot be observed regarding the mosquito circulatory system. Because of the multi-functional importance of haemolymph flow, studies focused on deciphering the mosquito circulatory system may provide a better understanding of the interactions between different physiological systems and the biology of pathogens as they migrate through the haemocoel (Andereck et al., 2010; Glenn et al., 2010).

Details of the mosquito heart morphology and function have been recently described for An. gambiae (Andereck et al., 2010; Glenn et al., 2010). These studies demonstrated the route taken by haemolymph as it enters the mosquito heart, and how abdominal contractions potentiate extracardiac haemolymph propulsion. In contrast, our assessment of the heart of several other mosquito species aimed at revealing architectural details for the study of heart morphology in these mosquito
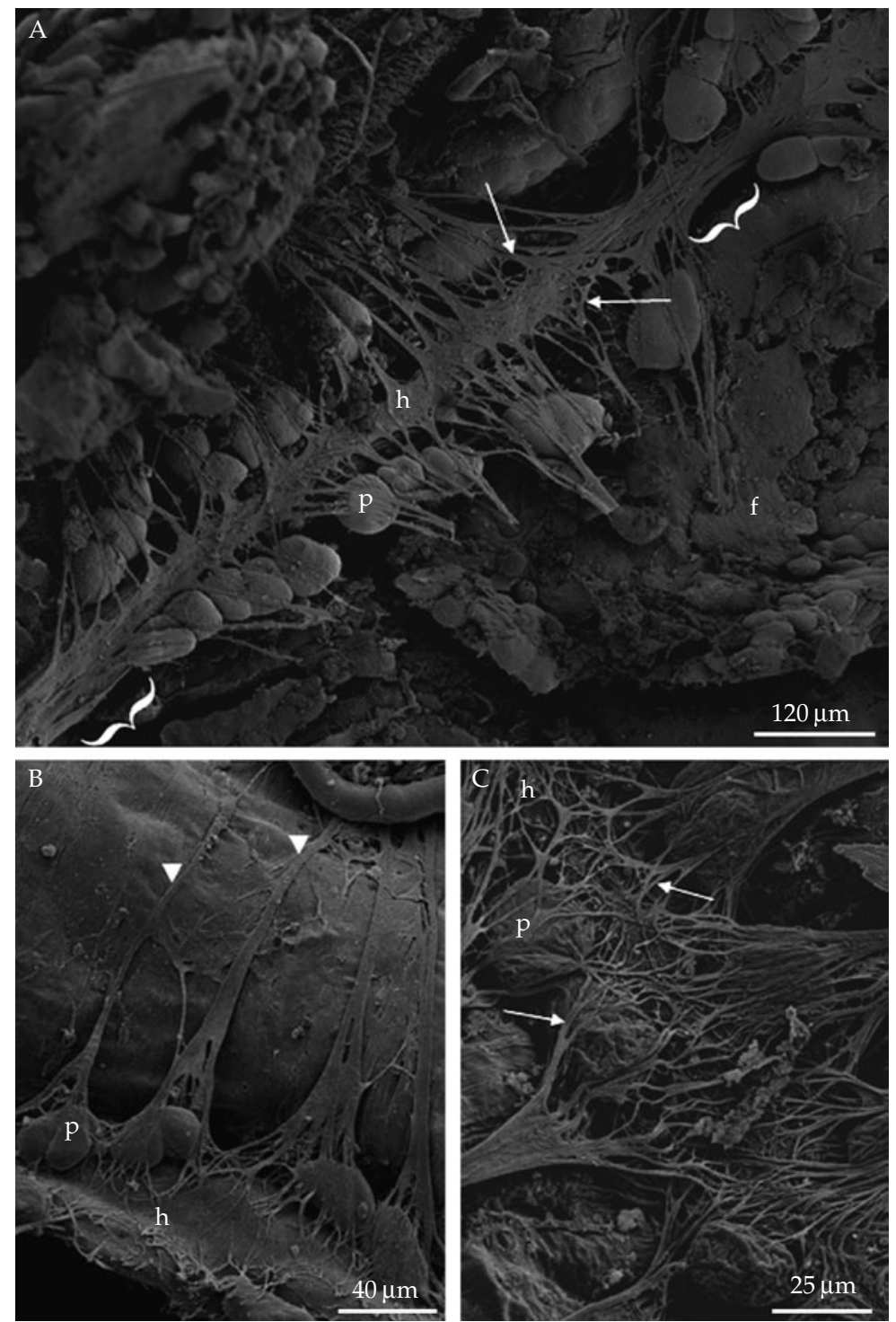

Fig. 1. SEM images showing the general and detailed ventral views of the heart (h) of mosquito. A, ventral view of the heart of Anopheles darlingi female. Arrows, alary muscles; f, fat body; p, pericardial cell; curly brackets, heart segments where pericardial cells are absent. B, alary muscles of the Aedes fluviatilis female are attached to the body wall (arrowheads) and connected to the heart. C, detail of the heart of the Aedes fluviatilis female, with a pericardial cell positioned on the edge of the heart and the alary muscles with their ramifications above the heart surface (arrows). 

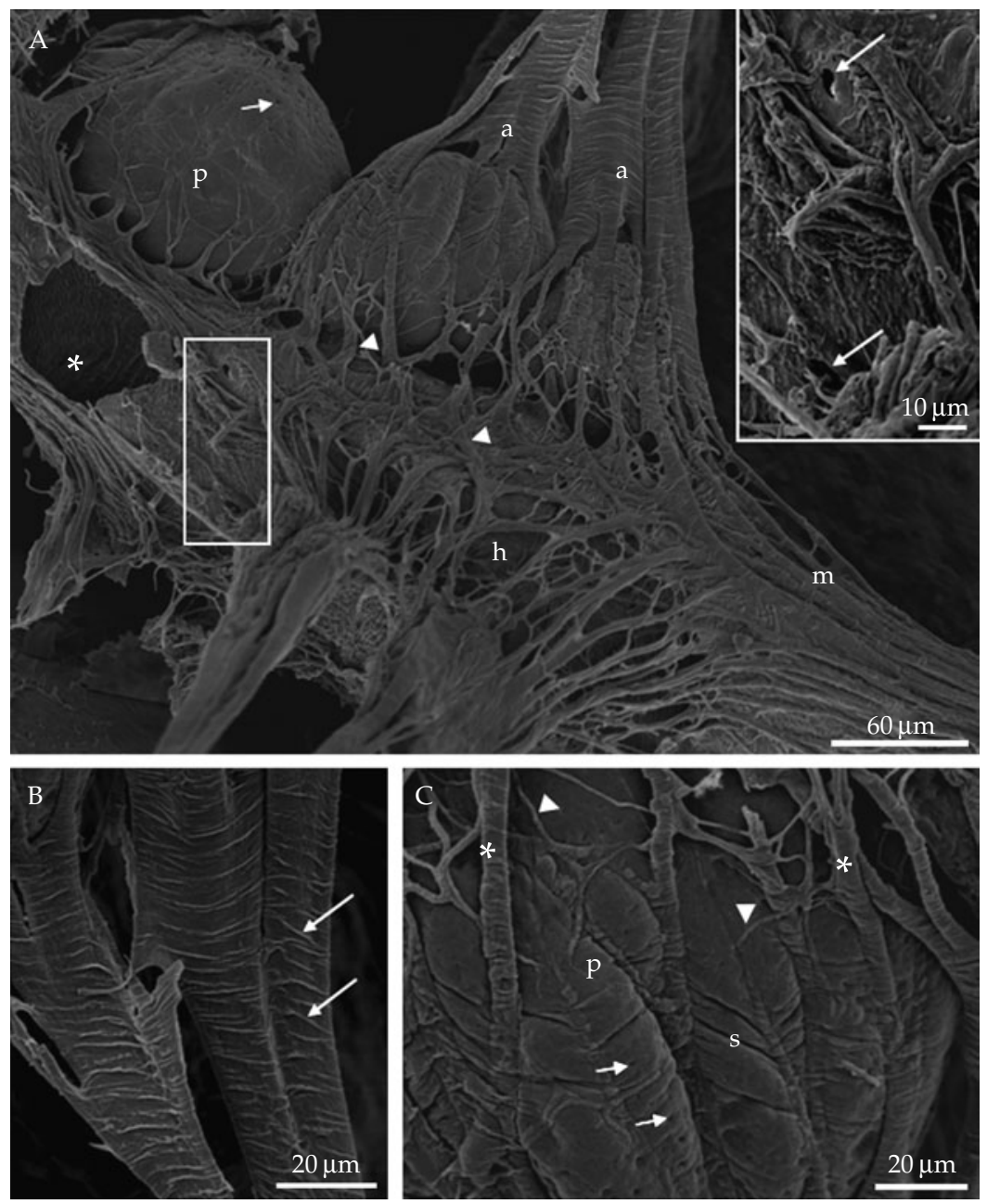

Fig. 2. SEM images showing details of the heart of Aedes aegypti female. A, paired alary muscles (a) with anastomosed prolongations (arrowheads) on the heart surface. * indicates the circular striated aspect of the heart muscle; the alary muscle myofibrils are shown extending throughout the heart surface $(\mathrm{m})$. p, pericardial cell; small arrows, pericardial cell invagination. Inset, ostia or pair of openings (arrows) where haemolymph is collected. B, alary muscles with striations (arrows). C, portion of pericardial cell that extrudes from the alary muscle ramifications (asterisks). Note the cell membrane invaginations (small arrows) and striations (s) on the pericardial cell surface that probably result from the compression of the pericardial cell against the alary muscle myofibrils (arrowheads).

species. Our studies were based on microscopic observations using SEM.

Not surprisingly, for the six adult mosquito species investigated, and irrespective of their sex, the heart was found to have the same general localization and basic morphological aspect. In this sense, the heart of mosquito consists of a muscular tube located directly below the dorsal cuticle with alary muscles and pericardial cells along the sides as described for other insects (Crossley, 1985; Miller, 1985; Curtis et al., 1999). In addition, the helical orientation of the heart muscle fibres was shown to be conserved between the mosquito species investigated in this study, and in agreement with the data reported for Anopheles spp. (Jones, 1954; Andereck et al., 2010; Glenn et al., 2010).
The mosquito alary muscles are believed to provide a physical support for the heart, maintaining its integrity (Jones, 1954; Andereck et al., 2010; Glenn et al., 2010). Accordingly, our results show that the alary muscles firmly bind to the ventral half of the heart and may constitute a suspensory apparatus. These muscles have a striated aspect and ramify forming a network (or basket) near the heart, which also provides an attachment site for the pericardial cells. In addition, alary muscle myofibrils appear to exert pressure on the surface of the pericardial cells leading to deformities.

In this study, we provided comparative morphological analyses of the hearts of several mosquito species significantly adding to the current available data. For these six distantly related 
mosquito species, the assessment of the heart architecture revealed a high level of conservancy with regard to their morphology. We believe that a comprehensive understanding of the heart morphology of mosquito vectors will contribute to future studies focused on various functional aspects of this organ and possibly lead to novel strategies in the fight against these important disease vectors.

\section{Acknowledgements}

This study was supported by Conselho Nacional de Desenvolvimento Científico e Tecnológico (CNPq), Fundação de Amparo a Pesquisa de Minas Gerais (Fapemig) and Fundação Oswaldo Cruz (Fiocruz).

\section{References}

Andereck J. W., King J. G. and Hillyer J. F. (2010) Contraction of the ventral abdomen potentiates extracardiac retrograde hemolymph propulsion in the mosquito hemocoel. PLoS One 5, e12943.

Chapman R. F. (ed.) (1998) The Insects. Structure and Function. Cambridge University Press, Cambridge. $788 \mathrm{pp}$.

Christophers S. R. (ed.) (1960) Aedes aegypti (L.), Yellow Fever Mosquito: Its Life History, Bionomics, and Structure. Cambridge University Press, Cambridge. 737 pp.

Crossley A. C. (1985) Nephrocytes and pericardial cells, pp. 487-515. In Comprehensive Insect Physiology, Biochemistry and Pharmacology, Vol. 3 (edited by G. A. Kerkut and L. Gilbert). Pergamon Press, Oxford.

Curtis N. J., Ringo J. M. and Dowse H. B. (1999) Morphology of the pupal heart, adult heart, and associated tissues in the fruit fly, Drosophila melanogaster. Journal of Morphology 40, 225-235.

Das D., Aradhya R., Ashoka D. and Inamdar M. (2008) Post-embryonic pericardial cells of Drosophila are required for overcoming toxic stress but not for cardiac function or adult development. Cell Tissue Research 331, 565-570.

Denholm B. and Skaer H. (2009) Bringing together components of the fly renal system. Current Opinion in Genetics and Development 19, 526-532.

Ejaz A. and Lange A. B. (2008) Peptidergic control of the heart of the stick insect, Baculum extradentatum. Peptides 29, 214-225.

Foster W. A. and Walker E. D. (2009) Mosquitoes (Culicidae), pp. 201-254. In Medical and Veterinary Entomology (edited by G. R. Mullen and L. A. Durden). Elsevier, Amsterdam/Boston/Heidelberg/London/ New York/Oxford/Paris/San Diego/San Francisco/ Singapore/Sydney/Tokyo.

Glenn J. D., King J. G. and Hillyer J. F. (2010) Structural mechanics of the mosquito heart and its function in bidirectional hemolymph transport. Journal of Experimental Biology 213, 541-550.

Jones J. C. (1954) The heart and associated tissues of Anopheles quadrimaculatus Say (Diptera: Culicidae). Journal of Morphology 94, 71-123.

Miller T. A. (1985) Structure and physiology of the circulatory system, pp. 289-353. In Comprehensive Insect Physiology, Biochemistry and Pharmacology, Vol. 3 (edited by G. A. Kerkut and L. Gilbert). Pergamon Press, Oxford.

Weavers H., Prieto-Sánchez S., Grawe F., Garcia-López A., Artero R., Wilsch-Braeuninger M., Ruiz-Gómez M., Skaer H. and Denholm B. (2009) The insect nephrocyte is a podocyte-like cell with a filtration slit diaphragm. Nature 457, 322-326. 